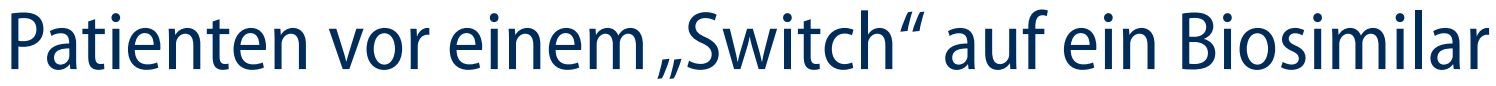 umfassend aufklären
}

Biosimilars erobern die Rheumatologie. Beim TNFa-Blocker Adalimumab liegt deren Anteil bereits bei $36 \%$.

Bei der Behandlung von Patienten aus dem rheumatischen Formenkreis kann seit einiger Zeit auf kosteneffizientere Biosimilar-Präparate zurückgegriffen werden. „Studien zeigen, weder in puncto Wirksamkeit noch hinsichtlich des Auftretens unerwünschter Ereignisse Unterschiede etwa zwischen dem AdalimumabOriginatorpräparat und dem Biosimilar Hulio ${ }^{\oplus ", ~ v e r d e u t l i c h t e ~ P r o f . ~ D r . ~}$ Christoph Baerwald, Leiter der Sektion Rheumatologie/Geriatrie am Universitätsklinikum Leipzig.

„Um ein Biosimilar in der Indikation des Originators verwenden zu können, wird dessen therapeutische Äquivalenz hinsichtlich Pharmakokinetik, Wirksamkeit, Verträglichkeit und Sicherheitsprofil umfassend in präklinischen und klinischen Phase-1- und Phase-3-Studien nachgewiesen", so der Rheumatologe beim Kongress der Deutschen Gesellschaft für Rheumatologie. Seit fast einem Jahr sind Adalimumab-Biosimilars wie etwa $\mathrm{Hulio}^{\circledR}$ für die Therapie bei Erkrankungen des rheumatischen Formenkreises, wie der rheumatoiden Arthritis, Psoriasis-Arthritis und axialen Spondylarthritis, in Deutschland erhältlich. Sie hätten sich in kürzester Zeit am Markt etabliert, sagte Baerwald. Allein in der ersten Jahreshälfte 2019 entfielen 36,3 \% aller Adalimumab-Verordnungen auf Biosimilar-Produkte [1].

\section{Nocebo-Effekte vermeiden}

Aus wissenschaftlicher Sicht könne der Wechsel zu einer Biosimilar-Therapie bedenkenlos erfolgen, betonte Baerwald. „Um aber Nocebo-Effekte zu vermeiden, sollte der Patient vor Beginn der Behandlung umfassend über die Unbedenklichkeit des Biosimilars aufgeklärt werden." Gerade wenn Patienten bereits lange Zeit mit einem Originator-Präparat behandelt worden sind, fällt ihnen die Umstellung oftmals schwer. Und so darf es nicht verwundern, wenn es dann zu erhöhten Abbruchraten nach dem Switch kommt, wie eine Studie jüngst feststellte [2].

„Wichtig ist, dass beim Patientengespräch der behandelnde Arzt selber von der Qualität von Biosimilars überzeugt ist", weiß Baerwald aus Erfahrung. Nur dann kann er auch den Patienten überzeugen. Denn der Patient nimmt nicht nur die Worte wahr, sondern auch Gestus, Mimik und Sprachfärbung wie jüngst ein Video-Experiment verdeutlichte [3].

\section{Bericht: Reimund Freye}

\section{Literatur:}

1. Arbeitsgemeinschaft Pro Biosimilars (2019) Biosimilars in Zahlen, Marktdaten Juni 2019, https://probiosimilars.de/img_upload/2019/08/Marktdaten-Biosimilars 1.Halbjahr-2019_.pdf?ddl=1. Zugegriffen: 19. Aug. 2019

2. Bakalos G et al (2019) Clin Ther. 41(1):15573

3. Gasteiger C et al (2019) Arthritis Care Res, on press. https://doi.org/10.1002/acr.24012

rheuma plus $2019 \cdot 18: 204$ https://doi.org/10.1007/s12688-01900296-4

(c) Springer-Verlag GmbH Austria, ein Teil von Springer Nature 2019

Hier steht eine Anzeige.

\section{算 Springer}

